\title{
The influence of group membership on cross-contextual imitation
}

\author{
Oliver Genschow $^{1} \cdot$ Simon Schindler ${ }^{2}$
}

Published online: 2 December 2015

(C) Psychonomic Society, Inc. 2015

\begin{abstract}
Research on mimicry has demonstrated that individuals imitate in-group members more strongly than outgroup members. In the present study, we tested whether such top-down modulation also applies for more extreme forms of direct mapping, such as for cross-contextual imitation settings, in which individuals imitate others' movements without sharing a common goal or context. Models on self-other control suggest that top-down modulations are based merely on a direct link between social sensory processing and imitation. That is, perceived similarities between oneself and another person is sufficient to amplify a shared representation between own and others' actions, which then trigger imitation. However, motivational accounts explain such findings with the assumption that individuals are motivated to affiliate with others. Because imitation is linked to positive social consequences, individuals should imitate in-group members more strongly than out-group members. We tested these two theoretical accounts against each other by applying a crosscontextual imitation paradigm. The results demonstrate that in-group members are more strongly cross-contextually imitated than out-group members the higher individuals' motivation to affiliate with the in-group is. This supports motivational models but not self-other control accounts. Further theoretical implications are discussed.
\end{abstract}

Keywords Imitation · Top-down modulation · Group influences

Oliver Genschow

Oliver.Genschow@Ugent.be

1 Department of Experimental Psychology, Ghent University, Henri Dunatlaan 2, 9000 Gent, Belgium

2 University of Kassel, Kassel, Germany
Imagine yourself being in a conversation with one of your friends. You are drinking a beer and are talking about the recent developments in your job as you suddenly realize that you are sitting in the same position as your friend: Both of you have your legs crossed and are leaning backwards in the chairs. In the literature, such engagement has been linked to mimicry (for an overview, see Chartrand \& Dalton, 2009) or imitation (for an overview, see Heyes, 2011). Interestingly, the degree of mimicking or imitative behavior critically depends on social factors (Lakin \& Chartrand, 2003; Lakin, Chartrand, \& Arkin, 2008; Leighton, Bird, Orsini, \& Heyes 2010b; Wang \& Hamilton 2012; Wang \& Hamilton 2015). Past research has put forward two different models to explain such social topdown influences: models on self-other control (Sowden \& Shah, 2014; Teufel, Fletcher, \& Davis, 2010) and motivational models (Chartrand \& Dalton, 2009; Wang \& Hamilton 2012). Going one step further, in the present research we tested whether group-membership of the target person moderates imitative behavior in an extreme form of direct mapping - that is, in a cross-contextual imitation setting, in which individuals imitate another person without sharing a common goal and context. Crucially, within this paradigm we tested the predictive validity of the two models on top-down influences against each other.

\section{Mimicry and automatic imitation}

Mimicry is usually defined as individuals' tendency to imitate others. It has been suggested that such mimicking behavior takes place non-consciously in the sense that individuals are not aware of their mimicking behavior (Chartrand \& Bargh, 1999; Chartrand \& Dalton, 2009). Within mimicry research, researchers distinguished between the mimicry of a wide range of different behaviors such as facial expressions 
(Bourgeois \& Hess, 2008; Dimberg, 1982). characteristics of language (Cappella \& Planalp, 1981; Giles \& Powesland, 1975; Webb, 1969, 1972). postures (LaFrance, 1982). gestures (Bernieri, 1988). or simple movements (Chartrand \& Bargh, 1999). Mimicry most often has been studied by social psychologists, using naturalistic paradigms that usually measure the frequency of mimicking acts in interactions between a participant and a confederate (Chartrand \& Bargh, 1999; Lakin et al., 2008; Stel, Blascovich, et al., 2010; Stel, van Baaren, et al., 2010; van Baaren, Janssen, Chartrand, \& Dijksterhuis, 2009; van Baaren, Horgan, Chartrand, \& Dijkmans, 2004). In contrast, research in cognitive psychology focuses on automatic imitation, which can be defined as the laboratory model of mimicry (Heyes, 2011). In an exemplary experiment, participants respond with two different movements to two different symbolic cues while seeing either a congruent or incongruent movement on a computer screen. Research has shown that in such a conflict task, individuals respond faster and with fewer errors to congruent, compared to incongruent trials (Brass, Bekkering, \& Prinz, 2001; Brass, Bekkering, Wohlschläger, \& Prinz, 2000). "Automatic," in this context, refers to the fact that participants' movements are influenced by the observed movements, although their responses are directed to the independent cues on the screen. Interestingly, and in contrast to mimicry, individuals are aware of the other movement's influence (Heyes, 2011). Less important for our research and different from mimicry and automatic imitation are conscious imitation paradigms, in which participants are - in contrast to mimicry and automatic imitation paradigms - explicitly instructed to imitate another person (Leighton, Bird, \& Heyes 2010a; Losin, Cross, Iacoboni, \& Dapretto, 2014).

Despite the slightly different phenomena and research methods, theoretical accounts on mimicry as well as those on automatic imitation assume similar processes. It is generally agreed that automatic imitation and mimicry are based on a direct mapping of observed and executed actions. For example, Ideomotor Theory (Greenwald, 1970; Prinz, 1990, 1997) - a theory shaped in cognitive psychology_proposes that the observation of an action evokes the same representation as its execution. This common representation then eventually triggers the execution of the observed action. Similarly, social psychologists put forward the so-called perception-behavior link to explain mimicry (Chartrand \& Bargh, 1999; Chartrand, Maddux, \& Lakin, 2005; Dijksterhuis \& Bargh, 2001). Such a link assumes that merely perceiving a specific movement evokes the same representation as the execution of this movement, thus facilitating the execution of the perceived movement. Support for both of these theoretical models comes from neurophysiological research including singlecell recordings in monkeys (Di Pellegrino, Fadiga, Fogassi, Gallese, \& Rizzolatti, 1992). fMRI (Gazzola \& Keysers, 2009; Keysers \& Gazzola, 2010). or motor TMS (Catmur,
Walsh, \& Heyes, 2007; Fadiga, Fogassi, Pavesi, \& Rizzolatti, 1995). demonstrating that the observation and the execution of an action share similar motor areas.

Based on the idea that the observation of a movement elicits the same representation as its execution, research on cross-contextual imitation demonstrated that imitation also occurs across different contexts and goals (Genschow \& Florack, 2014; Genschow, Florack, \& Wänke, 2013). In a series of experiments, Genschow and colleagues (2013; 2014) led participants to taste a drink while watching either a cross-contextually compatible or incompatible drinkingmovement. Participants in the compatible movement condition watched a video of an athlete in a gym lifting a barbell. Participants in the incompatible movement condition watched the same athlete stretching the barbell. Watching the lifting movement activated the compatible movement increasing participants' drink intake, because they more often raised the cups to their mouth. In contrast, watching the stretching movement activated a drinking incompatible movement decreasing participants' arm movements towards the mouth resulting in a decreased drink intake. Ruling out the alternative explanation that this cross-contextual imitation effect is due to an abstract approach-avoidance priming, the authors found no effects on the evaluation of the drinks and only an effect on drink intake when participants were able to imitate the movements (e.g., drinking from cups) but not when participants were not able to imitate the movements (e.g., drinking from a tube). While such cross-contextual imitation is somehow different from automatic imitation and mimicry, it also shares similarities with both constructs. On the one hand, it is similar to automatic imitation as the imitation effect is defined as the difference in performance between congruent and incongruent movements. On the other hand, it is similar to mimicry as the underlying process takes place on an unconscious level in the sense that participants are not aware of their imitative response.

\section{Top-down modulations in mimicry and automatic imitation}

Despite different experimental approaches on automatic imitation and mimicry, all of the above-reviewed literature strongly indicates a direct link between action observation and action execution. However, recent findings suggest that this link may critically depend on social contexts (Lakin \& Chartrand, 2003; Lakin et al., 2008; Leighton et al. 2010b; Wang \& Hamilton 2012; Wang \& Hamilton 2015). For example, stronger automatic imitation effects have been found for human, compared with non-human actions (Liepelt \& Brass, 2010; Liepelt, Cramon, \& Brass, 2008). for individuals with low narcissistic traits (Hogeveen \& Obhi, 2013; Obhi, Hogeveen, Giacomin, \& Jordan, 2013). or for situations in which direct eye contact is present (Wang, Newport, \& 
Hamilton, A. F. d. C 2011a; Wang, Ramsey, \& Hamilton 2011b) - to name just a few examples. In line with these findings, social psychological research on mimicry found that ingroup members are more strongly mimicked than out-group members (Bourgeois \& Hess, 2008; Cheng \& Chartrand, 2003; Lakin et al., 2008; Mondillon, Niedenthal, Gil, \& Droit-Volet, 2007; Yabar, Johnston, Miles, \& Peace, 2006). For instance, Cheng and Chartrand (2003, Experiment 1) found that, compared with low self-monitoring individuals, high self-monitors mimicked a confederate's gestures more when they believed the confederate to be a peer. Relatedly, Lakin, Chartrand, and Arkin (2008; Experiment 2) showed that participants who are excluded by their in-group, and thus were motivated to affiliate with the in-group, mimicked a confederate who was an in-group member more than a confederate who was an out-group member.

An open question is whether such group modulations are also present for more extreme forms of direct mapping, such as in cross-contextual imitation settings, in which individuals imitate others' movements without sharing a common goal or context. In the past decade, different theoretical models on top-down modulations in imitation and mimicry have been developed that may suggest different answers to this question. On the one hand, recent models on self-other control suggest a direct link between social sensory processing and imitation (Sowden \& Shah, 2014; Teufel et al., 2010). Such models argue that the shared representation of own and others' actions, which is crucial for imitation to occur (Gazzola \& Keysers, 2009; Keysers \& Gazzola, 2010). is based on perceived similarities between oneself and the other person. If others are perceived to be similar to oneself, others' physical characteristics are included into the shared representation, whereas physical characteristics of dissimilar others are not included into the representation. Neurophysiological research (Brass, Derrfuss, \& Von Cramon, 2005; Brass, Ruby, \& Spengler, 2009; Spengler, von Cramon, \& Brass, 2009a, b) has shown that this process may be located within the temporoparietal junction (TPJ) and the medial prefrontal cortex (mPFC). Both areas are involved in processing Theory of Mind and empathy, which are known to facilitate imitative responses (Decety \& Lamm, 2007; Decety \& Sommerville, 2003; Sperduti, Guionnet, Fossati, \& Nadel, 2014; Van Overwalle, 2009). In terms of crosscontextual imitation, self-other control models would assume that in-group members should be more strongly imitated than out-group members if in-group members are perceived as more similar to oneself than out-group members. In other words, similarity between oneself and the target person should be the mediator between groupmembership and imitation. Thus, self-other control models would predict that the perceived similarity between oneself and the target person is more crucial for imitation than the group of the target person itself.
On the other hand, other models explain top-down modulations in imitation and mimicry by motivational processes (Chartrand \& Dalton, 2009; Wang \& Hamilton 2012). That is, based on findings that mimicking others goes in line with positive social consequences, such as that individuals are liked more strongly (Chartrand \& Bargh, 1999). feel closer to others (van Baaren et al., 2004). or receive more help from others (van Baaren, Holland, Steenaert, \& Van Knippenberg, 2003). it is suggested that imitation is a "strategic intervention to change the social world for selfadvancement" (Wang \& Hamilton 2012, p.2). It is argued that such a process is most likely implemented by the medial prefrontal cortex (mPFC; Wang \& Hamilton 2012). In line with the assumption of such motivational models, research has shown that the stronger an individuals' motivation is to affiliate with others, the more they mimic others (Lakin et al., 2008; Losin et al., 2014). As mentioned, when being excluded from the in-group, individuals tend to more strongly imitate this group than when they have been included in the group (Lakin et al., 2008; Experiment 2). In terms of cross-contextual imitation, motivational accounts would expect that, compared with the out-group, the in-group should be more strongly imitated the stronger individuals' motivation is to affiliate with the in-group. In other words, motivational accounts would define individuals' motivation to affiliate with the in-group as the moderator of the effect of group-membership on imitation.

\section{Current study}

In the current study we tested the influence of groupmembership on cross-contextual imitation. More specifically, we tested two process assumptions against each other - that is, self-other control models versus motivational accounts underlying cross-contextual imitation. On the one hand, self-other control models would predict increased cross-contextual imitation of in-group members compared with out-group members, if in-group members are perceived as more similar to oneself than out-group members. Furthermore, perceived similarity between oneself and the target person should be the mediator of this effect. That is, the perceived similarity between oneself and the target person is the crucial factor for increased imitation, rather than the group itself. On the other hand, motivational accounts would expect participants' motivation to affiliate with the in-group acting as moderator of group influences on cross-contextual imitation. That is, the effect of group-membership on imitation should increase with increased individuals' motivation to affiliate with the ingroup. To test these two models against each other, we conducted a cross-contextual imitation experiment, in which we introduced the model either as an in-group member or as an out-group member. Additionally, to test the underlying process assumptions of the two different models against each other, we 
assessed participants' perceived similarity with the model as well as participants' motivation to affiliate with the in-group.

\section{Method}

\section{Participants and design}

A total of 160 students (137 females) from the University of Mannheim (Germany) participated for course credit in the experiment. The age ranged from 18 to 44 years $(M=20.84$, $S D=3.20$ ). We applied a 2 (movement: compatible vs. incompatible) x 2 (group: in-group vs. out-group) betweensubjects design. In addition, we assessed participants' perceived similarity with the model (potential mediator) as well as participants' motivation to affiliate with the in-group (potential moderator) as additional continuous factors to test the different process assumptions derived from self-other control models and motivational models.

\section{Procedure}

The experiment was conducted within a series of other experiments. Statistical analyses indicated that the manipulations of the previous studies did not have any influences on the actual experiment. The procedure of the experiment was in line with the Genschow et al. $(2013,2014)$ studies. As a cover story, the experimenter told participants that they would have to taste a new sports drink that was designed for consumption during workouts. Furthermore, it was stated that in order to simulate the context of working out, a video of an athlete exercising with a barbell would be presented two times. To ensure that every participant had an equal level of thirst, participants first drank from a 300-ml plastic cup of water until they were not thirsty anymore. Afterwards, participants indicated basic demographic characteristics and then watched a video of an athlete exercising with a barbell two times. For the first video ( $24 \mathrm{~s}$ ), the participants were instructed to just watch the video. For the second video $(80 \mathrm{~s})$, the experimenter offered participants ten plastic cups, each with a capacity of $20 \mathrm{ml}$, of an icetea-flavored drink. To make sure that each cup contained the same amount of the beverage, the experimenter dispensed exactly $7 \mathrm{ml}$ of the drink into each cup with a pipette. The experimenter told participants that they were allowed to taste from as many cups as they wanted until the video finished. The videos were taken from the first-person perspective. In the compatible movement condition, the athlete was standing upright lifting a barbell from his waist to his chest. In the incompatible movement condition, the same athlete was laying on his back pushing the barbell up from his chest. In both videos, the athlete moved the barbell 16 times with the same rhythm.

To manipulate group membership of the athlete, he was introduced with a picture and a short $\mathrm{CV}$ before participants watched the videos. In the in-group condition, the athlete was introduced as a German student from the local university. In the out-group condition, the athlete was introduced as a Turkish man working in a fitness center.

After watching the video, participants evaluated the drink on four items as part of the coverstory ("I like this sports drink"; "The sports drink appeals to me"; "I would recommend this sports drink to a friend"; "I would like to buy this sports drink") on 7-point scales ( $1=$ not at all, $7=$ very much $)$. Crombach's Alpha for the composite score was $\alpha=0.95$.

To assess participants' perceived similarity with the athlete, we adapted the in-group bias measure of Castano, Yzerbyt, Paladino, and Sacchi (2002). participants indicated on 7-point scales $(1=$ not at all, $7=$ very much $)$ to what degree six personal characteristics ("sporty," "interested," "balanced," "likeable," "motivated," "open") apply to themselves similarly as to the athlete. To prepare data for analysis we computed a composite score of these items (Cronbach's $\alpha=0.73$ ).

To measure participants' motivation to affiliate with the ingroup - that is, the local university — we adapted the six-item identification scale from Castano and colleagues (2002) for our purposes. Participants answered the following six questions on 7-point scales $(1=$ not at all, 7 = very much $)$ : "To which degree do you feel like a student of this university?"; "How strongly do you identify yourself with this university?"; "How strongly does this university shape your daily life?"; "How strongly do you feel associated with this university?"; "Would you buy a souvenir (e.g., a t-shirt) of this university?"; "Would you indicate on a social media platform (e.g., Facebook) that you are studying at this university?" To prepare data for analyzes, we computed a composite score of this scale (Cronbach's $\alpha=0.81$ ).

At the end of the experiment, participants were probed for suspicion, debriefed, and dismissed. To measure the consumed portion of the drink, we counted how many cups the participants had drunk.

\section{Results}

None of the participants correctly guessed the purpose of the experiment. To test the two theoretical models against each other, we first analyzed the hypothesis derived from the selfother control models (Sowden \& Shah, 2014; Teufel et al., 2010) and then the predictions derived from the motivational accounts (Chartrand \& Dalton, 2009; Wang \& Hamilton 2012).

\section{Self-other control model hypothesis}

Self-other control models on imitation (Sowden \& Shah, 2014; Teufel et al., 2010) would assume that the perceived similarity mediates the effect of group-membership on 
imitation. That is, cross-contextual imitation should be larger for in-group members than for out-group members, because in-group members are perceived as more similar to oneself than out-group members. To test this hypothesis, we applied three analyses. First, we tested whether participants feel more similar to the athlete when he is introduced as an in-group member than when he is introduced as an out-group member. To test this assumption, we conducted a $t$-test for independent samples with the manipulated group-membership as independent variable and participants' perceived similarity with the athlete as dependent measure. Confirming the hypothesis, the result indicates that participants felt more similar to the athlete when he was introduced as an in-group member $(M=5.31, S D$ $=0.64)$ than when he was introduced as an out-group member $(M=4.95, S D=0.72), t(158)=3.25, p=0.001, d=0.53$.

Second, we tested in a regression analysis $\left(R^{2}=0.12\right)$ the effect of group-membership on cross-contextual imitation. We first z-standardized all continuous variables (Aiken \& West, 1996). As predictors, we entered the dummy-coded movement $(1=$ incompatible movement, $0=$ compatible movement) and the dummy-coded group ( $1=$ in-group, $0=$ outgroup) into the regression. The amount of used cups served as dependent measure. The regression yielded a main effect for movement indicating that participants used more cups while watching the compatible movement $(M=3.13, S D=1.21)^{1}$ compared with the incompatible movement $(M=2.49, S D=$ 1.14), $\beta=-0.26, t(159)=-3.53, p=0.001$. Also, the main effect for group was significant, indicating that participants used more cups when they believed they were observing an in-group member $(M=3.08, S D=1.36)$ compared with an out-group member $(M=2.54, S D=0.98), \beta=0.22, t(159)=$ $2.97, p=0.003$. However, in contrast to the prediction of selfother control models, the crucial interaction between group and movement did not reach significance, $\beta=-0.01, t(159)$ $=-0.07, p=0.945$. This suggests that the group itself may not influence cross-contextual imitation.

Third, we tested the effect of the assumed mediator of group-membership on imitation - namely perceived similarity between the athlete and the participants - with another multiple regression analysis $\left(R^{2}=0.07\right)$. As predictors, we entered the dummy-coded movement $(1=$ incompatible movement, 0 = compatible movement) and the perceived similarity with the athlete into the regression. Again, the main effect for movement was significant, $\beta=-0.27, t(159)=-3.46, p=0.001$. However, in contrast to the prediction of self-other control models, the main effect of perceived similarity was not significant, $\beta=-0.04, t(159)=-0.45, p=0.651$. The interaction term between similarity and movement was not a significant predictor for imitation either, $\beta=0.08, t(159)=0.74, p=$ 0.463 . This suggests that perceived similarity between

\footnotetext{
${ }^{1}$ To ease the interpretation we report here the amount of cups drunk instead of the z-transformed values.
}

participants and the athlete may not have any effects on cross-contextual imitation.

\section{Motivational account hypothesis}

Motivational accounts (Chartrand \& Dalton, 2009; Wang \& Hamilton 2012) would assume participants' motivation to affiliate with the in-group to be the moderator of group influences on imitation. That is, the effect of group-membership on imitation should be stronger the higher individuals' motivation to affiliate with the in-group is. To test this assumption, we ran a multiple regression analysis $\left(R^{2}=0.18\right)$. First, we $z$ standardized all continuous variables (Aiken \& West, 1996). The amount of used cups served as the dependent measure. As predictors we entered the dummy-coded movement $(1=$ incompatible movement, $0=$ compatible movement), the dummy-coded group ( $1=$ in-group, $0=$ out-group), participants' motivation to affiliate with the in-group, and all possible interactions between these variables. Figure 1 illustrates the results. The regression yielded a main effect for movement indicating that participants used more cups while watching the compatible movement $(M=3.13, S D=1.21)$, compared with the incompatible movement $(M=2.49, S D=1.14), \beta=-0.26$, $t(159)=-3.49, p=0.001$. Also, the main effect for group was significant, indicating that participants used more cups when they believed they were observing an in-group member $(M=$ $3.08, S D=1.36)$, compared with an out-group member $(M=$ $2.54, S D=0.98), \beta=0.22, t(159)=2.87, p=0.005$. The main effect of participants' motivation to affiliate with the in-group, as well as all two-way interactions did not reach significance, $\beta<0.10, t(159)<1.27, p>0.219$. However, more important and in line with the hypothesis derived from motivational accounts on imitation, the three-way interaction between observed movement, group, and participants' motivation to affiliate with the in-group was significant, $\beta=-0.34, t(159)=$ $-2.38, p=0.018$, indicating that the relationship between group and movement depended on participants' motivation to affiliate with the in-group.

To investigate this three-way interaction in more detail, we conducted additional regression analyses separately for the inand the out-group. For participants who believed to observe an in-group member, the regression again yielded a main effect for video, indicating that participants who watched the compatible movement $(M=3.40, S D=1.28)$ used more cups than participants who watched the incompatible movement $(M=$ $2.75, S D=1.28), \beta=-0.24, t(79)=-2.19, p=0.031$. The main effect of affiliation motivation was not significant, $\beta=$ $-0.11, t(79)=-1.04, p=0.303$. However, the interaction between movement and affiliation motivation was marginally significant, $\beta=-0.27, t(79)=-1.78, p=0.079$. As can be seen in Fig. 1, this indicates that participants tendentially cross-contextually imitated the movement of the in-group member more strongly the more they identified themselves 

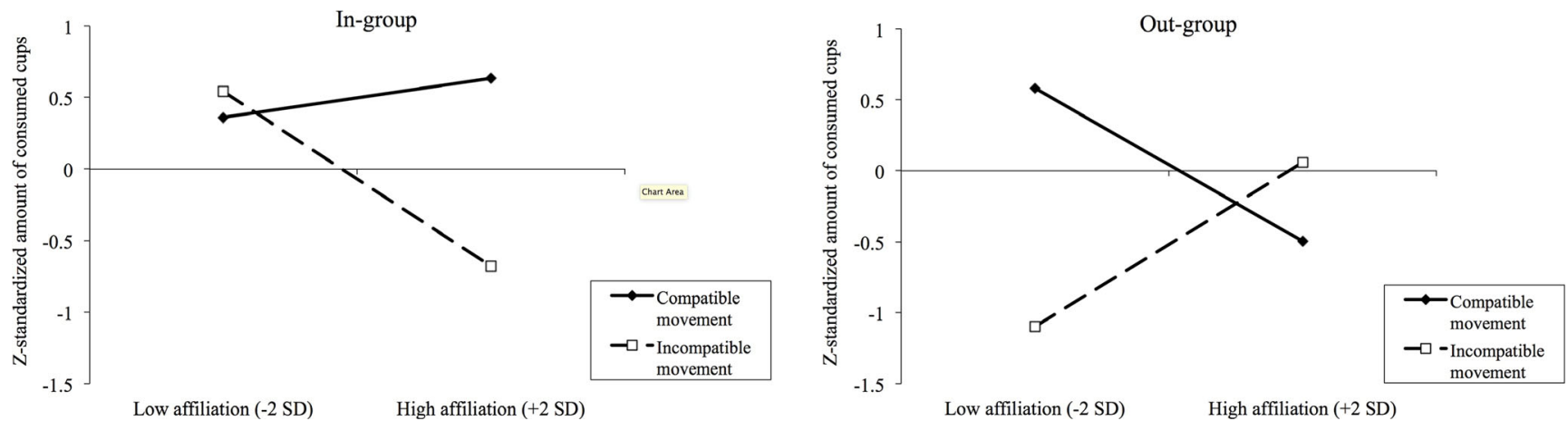

Fig. 1 Z-standardized number of cups drunk as a function of group (in-group vs. out-group), observed movement (incompatible vs. compatible), and motivation to affiliate with the in-group (low vs. high)

with the in-group. In addition, we ran simple slope analyses (Aiken \& West, 1996) for the two movement conditions. When participants watched the athlete executing the incompatible arm movement, participants used less cups the higher their affiliation motivation with the in-group was, $\beta=-0.32$, $t(79)=-2.02, p=0.047$. By contrast, when participants watched the athlete executing the compatible arm movement, affiliation motivation was not correlated with drink intake, $\beta=$ $0.07, t(79)=0.47, p=0.641$.

For participants who believed they were observing an outgroup member, the regression again yielded a main effect for video indicating that participants who watched the compatible movement $(M=2.85, S D=0.95)$ used more cups than participants who watched the incompatible movement $(M=2.23$, $S D=0.92), \beta=-0.32, t(79)=-2.93, p=0.004$. The main effect of participants' motivation to affiliate with the in-group was not significant, $\beta=-0.06, t(79)=-0.58, p=0.564$. Important for the hypothesis derived from motivational accounts on imitation, however, was the significant interaction between movement and affiliation motivation, $\beta=0.34, t(79)$ $=2.60, p=0.011$. As shown in Fig. 1, this indicates that participants cross-contextually imitated the movement of the out-group member less strongly the higher their motivation to affiliate with the in-group was. In addition, we ran simple slope tests (Aiken \& West, 1996) for the two movement conditions. When participants watched the athlete executing the incompatible arm movement, participants used marginally more of the cups the higher their affiliation motivation with the in-group was, $\beta=0.29, t(79)=1.70, p=0.093$. Conversely, when participants watched the athlete executing the compatible arm movement, affiliation motivation was negatively correlated with the number of cups used, $\beta=-0.27, t(79)=$ $-2.06, p=0.043$.

\section{Drink evaluation}

In additional multiple regression analyses, we tested whether the manipulations had an effect on the drink evaluation. In doing so, we ran all the above-reported analysis again with the reported drink evaluation as dependent variable. As in previous research on cross-contextual imitation (Genschow et al., 2013). neither a main effect nor any interaction predicted the drink evaluation, $\beta<0.11, t(159)<1.33, p>0.18$.

\section{Discussion}

In the current study, we tested the influence of groupmembership on cross-contextual imitation by comparing two process assumptions against each other. Self-other control models (Sowden \& Shah, 2014; Teufel et al., 2010) would have assumed that an in-group member is perceived as more similar to oneself than an out-group member and that this perceived similarity mediates the effect of groupmembership on cross-contextual imitation. The results show that an in-group member is, indeed, perceived as more similar than an out-group member. However, we did not find any support for the hypothesis that perceived similarity actually mediates the effect of group-membership on cross-contextual imitation for two reasons. First, group membership itself did not have any effect on cross-contextual imitation. Second, neither perceived similarity itself nor the interaction between perceived similarity and group membership had an effect on cross-contextual imitation. However, the data nicely fit motivational accounts underlying top-down influences in imitation (Chartrand \& Dalton, 2009; Wang \& Hamilton 2012). Motivational accounts would have expected that participants' motivation to affiliate with the in-group moderates the influence of group-membership on imitation. In line with this prediction, the results demonstrate that the effect of groupmembership on cross-contextual imitation is stronger the higher individuals' motivation to affiliate with the in-group is: When observing an in-group member, imitation of the model's movements increased with increasing motivation to affiliate with the in-group. Conversely, when observing an out-group member, imitation decreased with an increasing affiliation motivation. 
This finding nicely fits social psychological research showing that being excluded from the in-group leads individuals to more strongly mimic their in-group (Lakin, Chartrand, \& Arkin, 2008). It has been assumed that social exclusion evokes a strong motivation to affiliate, thus, increasing imitative responses. Further support for such an affiliationmotivation process comes from studies on emotional facial mimicry conducted by Bourgeois and Hess (2008). In their studies, the researchers found that happiness was mimicked regardless of group-membership and anger was not mimicked in out-group members. The researchers explain this finding with the fact that happiness, but not anger, is generally perceived as highly affiliative (Hess, Blairy, \& Kleck, 2000; Knutson, 1996). Moreover, most recent research suggests that in some cases even out-group members might elicit stronger automatic imitation effects when participants are motivated to affiliate with that group (i.e., when sharing implicit motivation goals; Rauchbauer, Majdandžić, Hummer, Windischberger, \& Lamm, 2015). In sum, these and our present findings suggest that affiliation motives are an important factor when it comes to imitation and mimicry of different group members.

Although the finding that affiliation motives are a key driver in cross-contextual imitation of groups, an intriguing question is why group membership should have an influence on cross-contextual imitation at all. Based on the fact that in cross-contextual imitation settings individuals cannot expect any positive social consequences, it might be rather surprising that affiliation motives and group membership still have a strong impact. A promising explanation for this finding is offered by research conducted by Losin et al. (2014; see also Losin, Iacoboni, Martin, Cross, \& Dapretto, 2012). The researchers conducted an fMRI study with European American and African American participants. Participants consciously imitated and passively observed gestures from other African American, European American, as well as Chinese American models. Within both African American and European American subjects, more activity in the lateral frontoparietal and visual regions was found when imitating African American models compared to European American or Chinese American models. Furthermore, it was found that African and European American subjects associated African Americans with lower social status than European or Chinese Americans. In line with our research, these findings suggest that it is not the perceived similarity between group and observer that modulates imitation. Instead, by taking an evolutionary perspective, Losin and colleagues speculate that neural responses towards groups during imitation might be driven by socially learned associations. That is, stronger imitation might reflect an adaptive way of social learning, in which learning from higher status models is more beneficial than learning from lower status models. An open question is whether similar processes are involved in cross-contextual imitation of in- and out-group members. Future research may aim at further exploring whether overlearned imitation of high status individuals transfers to imitative settings, such as cross-contextual imitation, in which no positive social consequences can be expected.

Despite the theoretical implications of our findings, there might be some shortcomings regarding the results. A close look at the interaction pattern suggests that the effects are driven by the compatible as well as by the incompatible movement condition. More specifically, simple slope tests suggest that when observing the in-group member the interaction between affiliation and observed movement is driven by the compatible movement condition. In contrast, the same interaction for observing the out-group member seems to be driven by the incompatible as well as by the compatible movement condition. It is important to note, however, that past research on automatic imitation (e.g., Brass et al., 2001; Brass et al., 2000) indicates that in some cases incompatible movements can indeed drive imitation effects. This is the case because the observation of any kind of movement activates corresponding motor programs (Brass et al., 2001; Kilner, Paulignan, \& Blakemore, 2003). Therefore, the observation of a compatible drinking-movement facilitates drinking resulting in an increased amount of drinking-movements whereas the observation of an incompatible drinking-movement can inhibit its execution resulting in a decreased amount of drinking-movements. Crucial for a cross-contextual imitation effect, thus, is not the effect of single slopes, but rather the difference between compatible and incompatible movements, which is the case in our study.

In sum, our findings suggest that despite any direct social advantages, shared goals and contexts, group-membership has still a strong impact on imitation. However, an important precondition for such group-modulations to occur is the motivation to affiliate with the in-group. This result supports motivational models on top-down influences in imitation (Chartrand \& Dalton, 2009; Wang \& Hamilton 2012) and extends models on self-other control (Sowden \& Shah, 2014; Teufel et al., 2010).

\section{References}

Aiken, L. S., \& West, S. G. (1996). Multiple Regression testing and interpreting interactions. London: Sage Publications.

Bernieri, F. J. (1988). Coordinated movement and rapport in teacherstudent interactions. Journal of Nonverbal Behavior, 12, 120-138.

Bourgeois, P., \& Hess, U. (2008). The impact of social context on mimicry. Biological Psychology, 77, 343-352.

Brass, M., Bekkering, H., \& Prinz, W. (2001). Movement observation affects movement execution in a simple response task. Acta Psychologica, 106, 3-22.

Brass, M., Bekkering, H., Wohlschläger, A., \& Prinz, W. (2000). Compatibility between observed and executed finger movements: comparing symbolic, spatial, and imitative cues. Brain and Cognition, 44, 124-143. 
Brass, M., Derrfuss, J., \& Von Cramon, D. Y. (2005). The inhibition of imitative and overlearned responses: a functional double dissociation. Neuropsychologia, 43, 89-98.

Brass, M., Ruby, P., \& Spengler, S. (2009). Inhibition of imitative behaviour and social cognition. Philosophical Transactions of the Royal Society B: Biological Sciences, 364, 2359-2367.

Cappella, J. N., \& Planalp, S. (1981). Talk and silence sequences in informal conversations III: Interspeaker influence. Human Communication Research, 7, 117-132.

Castano, E., Yzerbyt, V., Paladino, M.-P., \& Sacchi, S. (2002). I belong, therefore, I exist: Ingroup identification, ingroup entitativity, and ingroup bias. Personality and Social Psychology Bulletin, 28, 135-143.

Catmur, C., Walsh, V., \& Heyes, C. (2007). Sensorimotor learning configures the human mirror system. Current biology, 17, 1527-1531.

Chartrand, T. L., \& Bargh, J. A. (1999). The chameleon effect: The perception-behavior link and social interaction. Journal of Personality \& Social Psychology, 76, 893-910.

Chartrand, T. L., \& Dalton, A. N. (2009). Mimicry: Its ubiquity, importance, and functionality. In E. Morales, P. M. Gollwitzer \& J. A. Bargh (Eds.), The psychology of action: Vol. 2. Mechanisms of human action (pp. 893-910): Oxford University Press.

Chartrand, T. L., Maddux, W. W., \& Lakin, J. L. (2005). Beyond the perception-behavior link: The ubiquitous utility and motivational moderators of nonconscious mimicry. In R. Hassin, J. Uleman, \& J. A. Bargh (Eds.), Unintended thoughts 2: The new unconscious (pp. 334-361). New York: Oxford University Press.

Cheng, C. M., \& Chartrand, T. L. (2003). Self-monitoring without awareness: Using mimicry as a nonconscious affiliation strategy. Journal of personality and social psychology, 85, 1170-1179.

Decety, J., \& Lamm, C. (2007). The role of the right temporoparietal junction in social interaction: how low-level computational processes contribute to meta-cognition. The Neuroscientist, 13, 580-593.

Decety, J., \& Sommerville, J. A. (2003). Shared representations between self and other: a social cognitive neuroscience view. Trends in Cognitive Sciences, 7, 527-533.

Di Pellegrino, G., Fadiga, L., Fogassi, L., Gallese, V., \& Rizzolatti, G. (1992). Understanding motor events: a neurophysiological study. Experimental Brain Research, 91, 176-180.

Dijksterhuis, A., \& Bargh, J. A. (2001). The perception-behavior expressway: Automatic effects of social perception on social behavior. Advances in experimental social psychology, 33, 1-40.

Dimberg, U. (1982). Facial reactions to facial expressions. Psychophysiology, 19, 643-647.

Fadiga, L., Fogassi, L., Pavesi, G., \& Rizzolatti, G. (1995). Motor facilitation during action observation: a magnetic stimulation study. Journal of neurophysiology, 73, 2608-2611.

Gazzola, V., \& Keysers, C. (2009). The observation and execution of actions share motor and somatosensory voxels in all tested subjects: single-subject analyses of unsmoothed fMRI data. Cerebral Cortex, 19, 1239-1255.

Genschow, O., \& Florack, A. (2014). Attention on the Source of Influence Reverses the Impact of Cross-Contextual Imitation. Journal of Experimental Psychology: Human Perception and Performance, 40, 904-907.

Genschow, O., Florack, A., \& Wänke, M. (2013). The power of movement: Evidence for context-independent movement imitation. Journal of Experimental Psychology: General, 142, 763-773.

Giles, H., \& Powesland, P. F. (Eds.). (1975). Speech style and social evaluation. London: Academic Press.

Greenwald, A. G. (1970). Sensory feedback mechanisms in performance control: With special reference to the ideo-motor mechanism. Psychological Review, 77, 73-99.

Hess, U., Blairy, S., \& Kleck, R. E. (2000). The influence of facial emotion displays, gender, and ethnicity on judgments of dominance and affiliation. Journal of Nonverbal Behavior, 24, 265-283.
Heyes, C. (2011). Automatic imitation. Psychological Bulletin, 137, 463 483.

Hogeveen, J., \& Obhi, S. S. (2013). Automatic imitation is automatic, but less so for narcissists. Experimental Brain Research, 224, 613-621.

Keysers, C., \& Gazzola, V. (2010). Social neuroscience: mirror neurons recorded in humans. Current biology, 20, 353-354.

Kilner, J., Paulignan, Y., \& Blakemore, S. (2003). An interference effect of observed biological movement on action. Current biology, 13, $522-525$.

Knutson, B. (1996). Facial expressions of emotion influence interpersonal trait inferences. Journal of Nonverbal Behavior, 20, 165-182.

LaFrance, M. (1982). Posture mirroring and rapport. In M. Davis (Ed.), Interaction rhythms: Periodicity in communicative behavior (pp. 279-298). New York: Human Sciences Press.

Lakin, J. L., \& Chartrand, T. L. (2003). Using nonconscious behavioral mimicry to create affiliation and rapport. Psychological Science, 14, 334-339.

Lakin, J. L., Chartrand, T. L., \& Arkin, R. M. (2008). I am too just like you - Nonconscious mimicry as an automatic behavioral response to social exclusion. Psychological Science, 19, 816-822.

Leighton, J., Bird, G., \& Heyes, C. (2010a). 'Goals' are not an integral component of imitation. Cognition, 114, 423-435.

Leighton, J., Bird, G., Orsini, C., \& Heyes, C. (2010b). Social attitudes modulate automatic imitation. Journal of Experimental Social Psychology, 46, 905-910.

Liepelt, R., \& Brass, M. (2010). Top-down modulation of motor priming by belief about animacy. Experimental Psychology, 57, 221-227.

Liepelt, R., Cramon, D., \& Brass, M. (2008). What is matched in direct matching? Intention attribution modulates motor priming. Journal of Experimental Psychology: Human Perception and Performance, 34, 578.

Losin, E. A. R., Cross, K. A., Iacoboni, M., \& Dapretto, M. (2014). Neural processing of race during imitation: Self-Similarity Versus Social Status. Human brain mapping, 35, 1723-1739.

Losin, E. A. R., Iacoboni, M., Martin, A., Cross, K. A., \& Dapretto, M. (2012). Race modulates neural activity during imitation. Neuroimage, 59, 3594-3603.

Mondillon, L., Niedenthal, P. M., Gil, S., \& Droit-Volet, S. (2007). Imitation of in-group versus out-group members' facial expressions of anger: A test with a time perception task. Social neuroscience, 2, 223-237.

Obhi, S. S., Hogeveen, J., Giacomin, M., \& Jordan, C. H. (2013). Automatic imitation is reduced in narcissists. Journal of Experimental Psychology: Human Perception and Performance, 40, 920-928.

Prinz, W. (1990). A common coding approach to perception and action. In O. Neumann \& W. Prinz (Eds.), Relationships between perception and action (pp. 167-201). Berlin: Springer-Verlag.

Prinz, W. (1997). Perception and action planning. European journal of cognitive psychology, 9, 129-154.

Rauchbauer, B., Majdandžić, J., Hummer, A., Windischberger, C., \& Lamm, C. (2015). Distinct neural processes are engaged in the modulation of mimicry by social group-membership and emotional expressions. Cortex.

Sowden, S., \& Shah, P. (2014). Self-other control: a candidate mechanism for social cognitive function. Frontiers in Human Neuroscience, 8 .

Spengler, S., von Cramon, D. Y., \& Brass, M. (2009a). Control of shared representations relies on key processes involved in mental state attribution. Human brain mapping, 30, 3704-3718.

Spengler, S., von Cramon, D. Y., \& Brass, M. (2009b). Was it me or was it you? How the sense of agency originates from ideomotor learning revealed by fMRI. Neuroimage, 46, 290-298.

Sperduti, M., Guionnet, S., Fossati, P., \& Nadel, J. (2014). Mirror Neuron System and Mentalizing System connect during online social interaction. Cognitive processing, 1-10. 
Stel, M. 1., Blascovich, J., McCall, C., Mastop, J., Van Baaren, R. B., \& Vonk, R. (2010). Mimicking disliked others: Effects of a priori liking on the mimicry-liking link. European Journal of Social Psychology, 40, 867-880.

Stel, M. 1., van Baaren, R. B., Blascovich, J., van Dijk, E., McCall, C., Pollmann, M. M., . . Vonk, R. (2010). Effects of a priori liking on the elicitation of mimicry. Experimental Psychology, 57, 412-418.

Teufel, C., Fletcher, P. C., \& Davis, G. (2010). Seeing other minds: attributed mental states influence perception. Trends in Cognitive Sciences, 14, 376-382.

van Baaren, R., Holland, R. W., Steenaert, B., \& Van Knippenberg, A. (2003). Mimicry for money: Behavioral consequences of imitation. Journal of Experimental Social Psychology, 39, 393-398.

van Baaren, R., Janssen, L., Chartrand, T. L., \& Dijksterhuis, A. (2009). Where is the love? The social aspects of mimicry. Philosophical Transactions of the Royal Society B: Biological Sciences, 364, 2381-2389.

van Baaren, R. B., Horgan, T. G., Chartrand, T. L., \& Dijkmans, M. (2004). The forest, the trees, and the chameleon: context dependence and mimicry. Journal of personality and social psychology, 86, 453459.

Van Overwalle, F. (2009). Social cognition and the brain: a meta-analysis. Human brain mapping, 30, 829-858.
Wang, Y., \& Hamilton, A. F. d. C. (2012). Social top-down response modulation (STORM): a model of the control of mimicry in social interaction. Frontiers in Human Neuroscience, 6, 1-10. doi: 10. 3389/fnhum.2012.00153

Wang, Y., \& Hamilton, A. F. d. C. (2015). Anterior medial prefrontal cortex implements social priming of mimicry. Social cognitive and affective neuroscience, 10, 486-493.

Wang, Y., Newport, R., \& Hamilton, A. F. d. C. (2011). Eye contact enhances mimicry of intransitive hand movements. Biology letters, 7-10.

Wang, Y., Ramsey, R., \& Hamilton, A. F. d. C. (2011). The control of mimicry by eye contact is mediated by medial prefrontal cortex. The Journal of neuroscience, 31, 12001-12010.

Webb, J. T. (1969). Subject speed rates as a function of interviewer behavior. Language \& Speech, 12, 54-67.

Webb, J. T. (1972). Interview synchrony: An investigation of two speech rate measures in an automated standardized interview. In B. Pope \& A. W. Siegman (Eds.), Studies in dyadic communication (pp. 115133). New York: Pergamon.

Yabar, Y., Johnston, L., Miles, L., \& Peace, V. (2006). Implicit behavioral mimicry: Investigating the impact of group membership. Journal of Nonverbal Behavior, 30, 97-113. 